\title{
Creation of Neutral Disk-like Zone Around the Active Hot Star in Symbiotic Binaries
}

\author{
Zuzana Cariková and Augustin Skopal \\ Astronomical Institute, Slovak Academy of Sciences, \\ 05960 Tatranská Lomnica, Slovakia \\ email: zcarikova@ta3.sk
}

\begin{abstract}
We investigated the ionization structure of symbiotic binaries during their active phases. We found that a neutral disk-like zone around the hot star can be created as a result of its enhanced wind and fast, $200-300 \mathrm{~km} \mathrm{~s}^{-1}$, rotation. Calculated column densities of the neutral hydrogen atoms throughout the neutral zone and emission measures of the ionized part of the wind from the hot star are in a good agreement with those derived from observations.
\end{abstract}

Keywords. Stars: binaries: symbiotic, stars: winds: outflows

\section{Introduction}

Symbiotic stars are long-period interacting binary systems, which comprise a late-type giant and a hot compact star, which is in most cases a white dwarf. Accretion from the giant's wind makes the white dwarf to be a strong source of ionizing radiation $\left(T \sim 10^{5} \mathrm{~K}\right)$. During so called quiescent phases, it ionizes a fraction of the giant's wind giving rise to a dense nebula extended within the binary. However, during active phases, the ionization structure changes significantly. Modeling the spectral energy distribution of symbiotic binaries with high orbital inclination indicated the presence of a two-temperature type UV spectrum (Skopal 2005). The cooler component is produced by a relatively warm stellar source $(T \sim 22000 \mathrm{~K})$, while the hotter one is represented by the highly ionized emission lines and a strong nebular continuum. Skopal (2005) explained this type of the UV spectrum by a model in which a neutral disk-like structure surrounds the accretor in the orbital plane and hot emitting regions are located above/below the disk. The rapid creation of such an ionization structure during the first days/weeks of outbursts is connected with the enhanced wind from the hot star. Due to the fast rotation of the accretor, the wind particles are compressed more to the equatorial plane, where they create a neutral zone in the form of a flared disk. We tested the latter by applying the wind compression model. The presence of such disks is transient, being connected with the active phases of symbiotic binaries.

\section{Ionization structure in the wind from the hot star}

Modeling of the broad $\mathrm{H} \alpha$ wings showed that the stellar wind from the hot star is significantly enhanced to $\sim 10^{-7}-10^{-6} \mathrm{M}_{\odot} \mathrm{yr}^{-1}$ during active phases. However, during quiescent phases, the mass loss rate of the hot star is $\sim 10^{-8} \mathrm{M}_{\odot} \mathrm{yr}^{-1}$ (Skopal 2006). Rotation of the hot star leads to compression of the outflowing material towards the equatorial plane due to the conservation of angular momentum. The mechanism is described by the so-called wind compression model, which was developed by Bjorkman \& Cassinelli (1993). If the streamlines of gas from both hemispheres do not cross the equatorial plane then we are talking about the wind compressed zone model described 


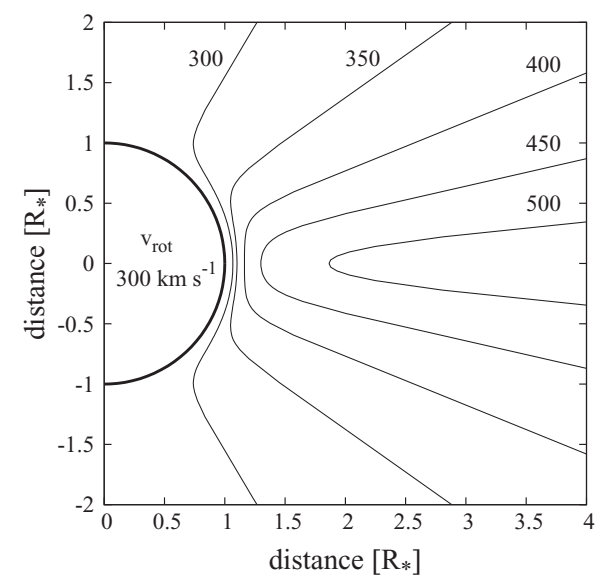

Figure 1. Calculated ionization boundaries in the stellar wind from the rotating hot star with the rotational velocity $300 \mathrm{~km} \mathrm{~s}^{-1}$. Individual ionization boundaries are denoted by the value of the parameter $X$. On the left from each ionization boundary there is the ionized zone and on the right there is the neutral zone. Distances are given in units of the radius of the hot star.

by Ignace et al. (1996). We calculated the ionization boundary in the compressed wind from the hot star using the equation for photoionization equilibrium. For simplicity, we assumed that the wind contains only hydrogen atoms. We calculated models for different rotational velocities of the hot star from 100 to $350 \mathrm{~km} \mathrm{~s}^{-1}$. Figure 1 shows some examples of the ionization boundaries which were calculated for the different values of the ionization parameter $X$ (given by the parameters of the hot star and its wind) resulting in the creation of the neutral disk-like zone near the equatorial plane of the hot star.

\section{Discussion and conclusion}

We showed that during active phases, the compression of the enhanced stellar wind from the rotating hot star towards the equatorial regions can lead to the creation of the neutral disk-like zone around the hot star. In symbiotic binaries with high orbital inclination, we are looking through the neutral disk-like zone up to a high optical depth, at which the material simulates a pseudophotosphere which radiates at significantly lower temperature than the hot central star. Further, we calculated column densities of the neutral hydrogen atoms in the disk-like zone above the pseudophotosphere and emission measures of the ionized part of the wind from the hot star, which are in a good agreement with the observed quantities. During quiescent phases, there is no neutral disk-like zone around the hot star, because of insufficient mass loss rate of the hot star.

\section{Acknowledgement}

This research was supported by a grant from the Slovak Academy of Sciences, VEGA No. 2/0038/10. Z. C. acknowledges support from the IAU.

\section{References}

Bjorkman, J. E. \& Cassinelli, J. P. 1993, ApJ, 409, 429

Ignace, R., Cassinelli, J. P., \& Bjorkman, J. E. 1996, ApJ, 459, 671

Skopal, A. 2005, A\&A, 440, 995

Skopal, A. 2006, $A \& A, 457,1003$ 